\title{
Neural Basis of Superior Performance of Action Videogame Players in an Attention-Demanding Task
}

\author{
Jyoti Mishra, ${ }^{1}$ Marla Zinni, ${ }^{2}$ Daphne Bavelier, ${ }^{3}$ and Steven A. Hillyard ${ }^{2}$ \\ ${ }^{1}$ Department of Neurology, University of California, San Francisco, San Francisco, California 94158, ${ }^{2}$ Department of Neurosciences, University of California, \\ San Diego, La Jolla, California 92093, and ${ }^{3}$ Brain and Cognitive Sciences Department and Center for Visual Science, University of Rochester, Rochester, New \\ York 14627
}

Steady-state visual evoked potentials (SSVEPs) were recorded from action videogame players (VGPs) and from non-videogame players (NVGPs) during an attention-demanding task. Participants were presented with a multi-stimulus display consisting of rapid sequences of alphanumeric stimuli presented at rates of $8.6 / 12 \mathrm{~Hz}$ in the left/right peripheral visual fields, along with a central square at fixation flashing at $5.5 \mathrm{~Hz}$ and a letter sequence flashing at $15 \mathrm{~Hz}$ at an upper central location. Subjects were cued to attend to one of the peripheral or central stimulus sequences and detect occasional targets. Consistent with previous behavioral studies, VGPs detected targets with greater speed and accuracy than NVGPs. This behavioral advantage was associated with an increased suppression of SSVEP amplitudes to unattended peripheral sequences in VGPs relative to NVGPs, whereas the magnitude of the attended SSVEPs was equivalent in the two groups. Group differences were also observed in the event-related potentials to targets in the alphanumeric sequences, with the targetelicited P300 component being of larger amplitude in VGPS than NVGPs. These electrophysiological findings suggest that the superior target detection capabilities of the VGPs are attributable, at least in part, to enhanced suppression of distracting irrelevant information and more effective perceptual decision processes.

\section{Introduction}

Videogame players (VGPs) have been found to exhibit superior visual performance on a variety of attention-demanding tasks, including spatial target localization, rapid target identification, and multi-object tracking (Green and Bavelier, 2003, 2006a,b, 2007). VGPs generally perform better than non-videogame player (NVGP) controls in tasks that require attention to visual displays that present high loads of information (Green and Bavelier, 2003) or dividing attention between multiple sources (Green and Bavelier, 2006b). More recently, it was shown that VGPs are also more resistant to attentional capture than NVGPs (Chisholm et al., 2010). Not all aspects of attention may be equally altered by videogame play, however. In particular, little or no gaming effect has been noted in attentional cueing (Castel et al., 2005; Dye et al., 2009), which suggests greater trainability of endogenous than exogenous forms of attention (for review, see Hubert-Wallander et al., 2010) (for another viewpoint, see West et al., 2008).

Both spatial and temporal aspects of top-down endogenous attentional selection have been demonstrated to improve as a result of action game play. Subjects trained on action videogames outperformed subjects trained on control games in tasks that engaged spatially selective attention (Green and Bavelier, 2003,

Received Sept. 14, 2010; revised Nov. 1, 2010; accepted Nov. 2, 2010.

This work was supported by Multidiciplinary University Research Initiative Grant N00014-07-1-0937 from the Office of Naval Research and National Institutes of Health Grants MH82790, MH86385, EY016984, and EY016880.

Correspondence should be addressed to Jyoti Mishra, University of California, San Francisco, Genentech Hall, Room N474, 600 16th Street, MC 2240, San Francisco, CA 94158. E-mail: jyoti@gazzaleylab.ucsf.edu.

DOI:10.1523/JNEUROSCI.4834-10.2011

Copyright $\odot 2011$ the authors $\quad 0270-6474 / 11 / 310992-07 \$ 15.00 / 0$ 2006b, 2007; Feng et al., 2007; Spence et al., 2009) (for an exception, see Boot et al., 2008) and in tasks that involved the temporal focusing of attention such as the attentional blink (Green and Bavelier, 2003; Cohen et al., 2007) (for an exception, see Boot et al., 2008) and backward masking (Li et al., 2010) paradigms. However, at present, little is known about the brain mechanisms that underlie these enhanced attentional capabilities of action game trainees.

The present study builds on these previous behavioral studies and introduces a paradigm that requires both spatial and temporal selection to investigate the neural bases of attentional superiority in VGPs. Participants were cued to attend to one of three rapid serial visual presentation (RSVP) sequences in the left (LVF), right (RVF), or central visual fields, each flashing at a different rate, with the task of detecting occasional targets in the attended sequence. Cortical processing of each flashing sequence was analyzed by recording its steady-state visual evoked potential (SSVEP), which is the frequency-tagged neural response of the visual cortex evoked by a periodic stimulus that can be recorded non-invasively from the scalp (Vialatte et al., 2010). Previous studies have demonstrated that the amplitude of the SSVEP is enhanced when attention is directed toward the driving stimulus (for review, see Di Russo et al., 2002; Toffanin et al., 2009). In a multistimulus display such as that used in the current experiment, the amplitude of the SSVEP to each concurrently flashing stimulus sequence provides a measure of the attentional resources being allocated to that stimulus (Müller et al., 2003; Toffanin et al., 2009). By recording attention-related modulations of SSVEPs and event-related potentials (ERPs) to target stimuli, we investigated how group differences in target detection per- 
A

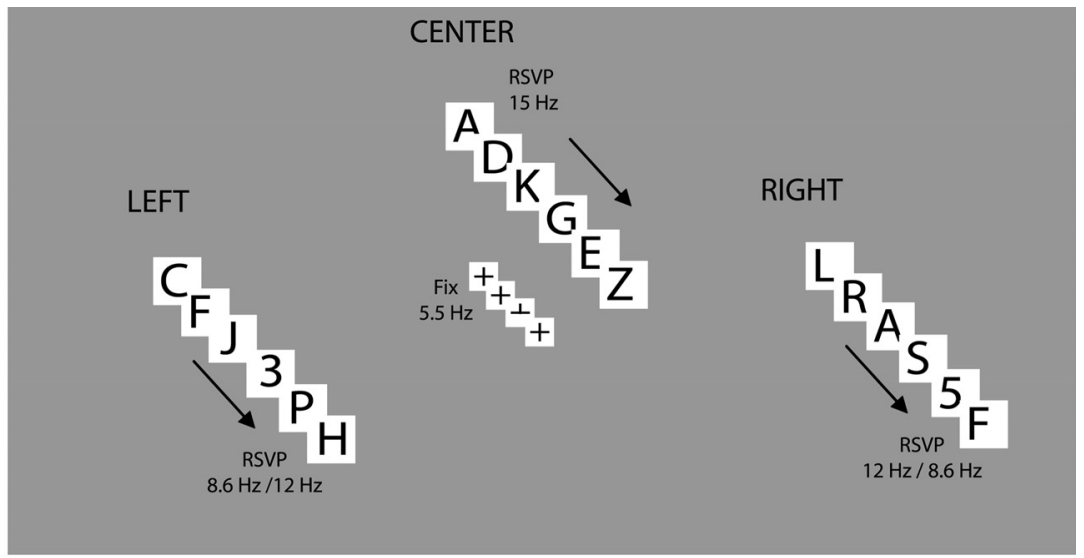

B

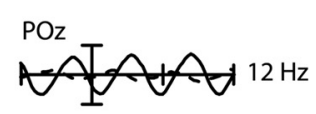

- - attended
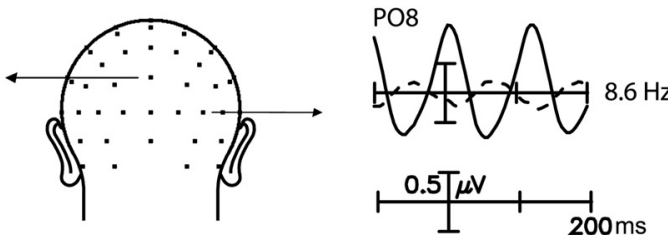

Figure 1. Overview of experimental design. $\boldsymbol{A}$, Rapid sequences of letters were presented at three peripheral visual field positions: left, right, and above fixation. Subjects maintained fixation on a central cross surrounded by a square flashing at $5.5 \mathrm{~Hz}$. On two-thirds of the trials subjects were cued to attend to the letter sequences in either the left or right visual field (flashing at 8.6 or 12 letters/s), with the task of detecting occasional numbers (targets) in the sequence. On the other one-third of the trials, subjects detected targets at fixation (disappearances of the lower arm of the cross). The $15 \mathrm{~Hz}$ letter sequence above fixation was always irrelevant and served to increase the information load in the display. $\boldsymbol{B}$, Time domain averages of SSVEPs to concurrent flashing letter sequences in the right $(12 \mathrm{~Hz})$ and left $(8.6 \mathrm{~Hz}$ ) visual fields recorded from occipital electrode sites POz and P08, respectively, in a typical participant. Because the SSVEP has the same fundamental frequency as the driving stimulus, it can be recorded separately to each of the concurrent stimulus sequences that are flashing at different rates. Solid waveforms correspond to attended trials and dashed waveforms to unattended trials, wherein attention was cued to the letter sequence in the opposite field.

formance between VGPs and NVGPs were related to cortical mechanisms of attention.

\section{Materials and Methods}

Subjects. Forty-one right-handed healthy adults, with normal or correctedto-normal vision, participated in the study after giving written informed consent as approved by the University of California, San Diego Human Research Protections Program. Participants were classified as belonging to one of two groups, VGPs and NVGPs, on the basis of a questionnaire about their videogame playing habits (Green and Bavelier, 2007). All participants in both groups were males because of the relative scarcity of female VGPs. To be considered a VGP, a subject needed to report a minimum of $5 \mathrm{~h} /$ week of action game play for the previous year $(n=21$; mean age, 21 years; mean action game play, $9.0 \pm 2.7 \mathrm{~h} /$ week; range, $5-12.5 \mathrm{~h}$ of game play uniformly distributed over the week). The criterion for inclusion in the NVGP group was a report of $0 \mathrm{~h} /$ week of action game play over the past year and little or no action gaming experience before that $(n=20$; mean age, 24 years). These gaming criteria were identical to those used in previous behavioral studies conducted on action videogame players in the Bavelier laboratory (Green and Bavelier, 2003, 2006a,b, 2007).

Stimuli and task. During testing, subjects sat $70 \mathrm{~cm}$ from a video monitor and were instructed to maintain visual fixation on a black cross that was centered inside a solid $0.8^{\circ}$ white square that flickered at a rate of 5.5 $\mathrm{Hz}$ (100 ms on, $83.3 \mathrm{~ms}$ off). Concurrent with this flashing central square, three letter sequences were delivered in RSVP to the LVF, RVF, and above fixation, respectively. The LVF and RVF sequences were presented at 8.6 $\mathrm{Hz}$ (50 ms on, $66.7 \mathrm{~ms}$ off) in one field and at $12 \mathrm{~Hz}$ ( $50 \mathrm{~ms}$ on, $33.3 \mathrm{~ms}$ off) in the other. These letter sequences in the LVF and RVF consisted of randomized presentations of 12 letters of the alphabet (A, D, E, G, J, K, $\mathrm{M}, \mathrm{N}, \mathrm{P}, \mathrm{R}, \mathrm{W}, \mathrm{Y}$ ) that were presented in black Arial font on a white square of $4^{\circ}$ size and $8^{\circ}$ eccentricity. The RSVP sequence above fixation was located at $7^{\circ}$ elevation, presented letters at $15 \mathrm{~Hz}(50 \mathrm{~ms}$ on, $16.7 \mathrm{~ms}$ off), and otherwise had the same parameters as the lateral letter stimuli. Infrequent numbers $(2,3$, and 5$)$ were interspersed at random in the lateral letter sequences and served as targets. Target probabilities were 0.031 and 0.022 per character for the 8.6 and $12 \mathrm{~Hz}$, sequences, respectively, so that a unique target $(2,3$, or 5$)$ occurred in each stimulus stream on average every $3.75 \mathrm{~s}$. Target randomization was constrained such that no two consecutive targets appeared $<2 \mathrm{~s}$ apart, thereby ensuring that successive targets did not occur within an attentional blink interval. The $15 \mathrm{~Hz}$ letter sequence above fixation had no interspersed number targets and served to increase the information load of the display. Targets at fixation were designated as infrequent disappearances of the lower arm of the fixation cross (occurring once every $5.7 \pm 1.7 \mathrm{~s}$ ). The experimental design is shown schematically in Figure 1.

The experiment consisted of eight blocks of $4.6 \mathrm{~min}$ each, with left/right placement of $8.6 / 12 \mathrm{~Hz}$ stimuli alternating in successive blocks and starting block order counterbalanced across subjects. Each block consisted of 24 trials of $8 \mathrm{~s}$ each, preceded by a $3.5 \mathrm{~s}$ cue. The centrally presented cue $(<,>$, or +$)$ directed subjects to attend to the peripheral alphanumeric sequence on either the left or right, or to attend to the central fixation cross targets, respectively, but ignore the other concurrent sequences. Each block contained eight trials per cue direction, presented in random order. Subjects responded to targets in the attended sequence by pressing a button on a response pad held in their lap and ignored targets in the unattended sequences. The $15 \mathrm{~Hz}$ letter stream in the upper field lacked targets and was never cued. Also, no central fixation targets were presented during trials when the peripheral sequences were attended; the left and right peripheral targets were always presented, however, even during trials when attention was directed to central fixation targets. Speed and accuracy were both emphasized in the behavioral task, and correct responses (hits) were scored within a $200-1000 \mathrm{~ms}$ period after target onset. Responses made outside of this target window were classified as "false alarms." The hit and false alarm rates were used to derive the sensitivity estimate $d^{\prime}$ (Macmillan and Creelman, 1991).

Electrophysiological recordings. The EEG was recorded from 62 electrode sites using a modified 10-10 system montage (Teder-Sälejärvi et al., 2005). Horizontal and vertical electro-oculograms (EOGs) were recorded by means of electrodes at the left and right external canthi and an electrode below the left eye, respectively. The importance of maintaining fixation was emphasized to participants, and the experimenter continually monitored the EOG and verified fixation in all blocks. All electrodes were referenced to the right mastoid electrode. Electrode impedances were kept below $5 \mathrm{k} \Omega$.

EEG and EOG signals were amplified with a gain of 10,000 and a bandpass of $0.1-80 \mathrm{~Hz}(-12 \mathrm{~dB} /$ octave; $3 \mathrm{~dB}$ attenuation $)$ and were digitized at $250 \mathrm{~Hz}$. Automated artifact rejection was performed before averaging to discard trials with eye movements, blinks, or amplifier blocking. Trials were rejected for eye movements or blinks when EOG deflections exceeded $\sim 50-75 \mu \mathrm{V}$. On average, $4 \%$ of trials were rejected over all subjects, with no significant difference in rejection rate between VGPs and NVGPs. Time-domain averages of the SSVEPs at each flicker frequency, as well as ERPs to targets were calculated offline. SSVEPs were averaged over successive $500 \mathrm{~ms}$ epochs that were centered at the onset of each stimulus in the sequence. These "moving window" averages were initiated $250 \mathrm{~ms}$ after the onset of each $8 \mathrm{~s}$ trial sequence and terminated $250 \mathrm{~ms}$ before the end of the sequence. Averages were digitally low-pass 
filtered with a Gaussian finite impulse function ( $3 \mathrm{~dB}$ attenuation at 46 $\mathrm{Hz}$ ) to remove high-frequency noise produced by muscle activity and external electrical sources. The filtered averages were digitally rereferenced to the average of the left and right mastoids. The magnitude of SSVEP activity at each stimulation frequency under each attention condition was quantified as the root mean square (rms) amplitude of the time-domain average.

The three-dimensional coordinates of each electrode and of three fiducial landmarks (the left and right pre-auricular points and the nasion) were determined by means of a Polhemus spatial digitizer. The mean Cartesian coordinates for each site were averaged across all participants and used for topographic mapping.

Data analysis. SSVEP amplitudes at each of the four stimulation frequencies were measured as the mean rms voltage over a specific cluster of electrodes at which the amplitudes at that frequency were maximal. The SSVEPs to the peripheral 8.6 and $12 \mathrm{~Hz}$ sequences were measured over 18 occipital electrode sites (nine in each hemisphere: P7/8, P5/6, P3/4, P1/2, $\mathrm{PO} / 8, \mathrm{PO} 3 / 4, \mathrm{O} 1 / 2, \mathrm{I} 3 / 4, \mathrm{I} 5 / 6$ ), whereas the SSVEPs to the central 5.5 and $15 \mathrm{~Hz}$ sequences were measured over nine occipital sites around the midline (PO3/4, O1/2, I3/4, POz, Oz, Iz). These SSVEP amplitude measures were subjected to between-group (VGPs vs NVGPs) repeatedmeasures ANOVAs that included factors of condition (attended vs unattended), location of stimulus presentation (left vs right) when applicable, and hemisphere of recording (left vs right).

For each participant, attended and unattended SSVEP amplitude measures were used to calculate an attentional modulation index (AMI) $=$ (attended - unattended) $/$ (attended + unattended). Correlations of AMI with behavioral measures of target detection accuracy $\left(d^{\prime}\right)$ and reaction time were calculated using the Pearson's correlation statistic.

The P300 component in the ERPs to targets was quantified over six parietal-occipital electrode sites (P1/P2, PO3/4, Pz, POz). The P300 amplitude was measured as the mean voltage over a $450-470 \mathrm{~ms}$ interval, which encompassed the peak in the grand-averaged waveforms, with respect to a $100 \mathrm{~ms}$ prestimulus baseline. Target ERPs were also averaged time-locked to the subjects' button-press responses, and P300 amplitudes were quantified in the $20 \mathrm{~ms}$ interval surrounding the peak in the response-locked grand-averaged waveforms. P300 amplitudes were compared between VGPs and NVGPs using one-tailed $t$ tests under the a priori hypothesis that VGPs would have larger amplitudes than NVGPs because of their superior performance.

Modeling of ERP sources. Inverse source modeling was performed to estimate the intracranial generators of the grand-averaged SSVEPs to the attended 8.6 and $12 \mathrm{~Hz}$ lateral stimuli in VGPs and NVGPs. Source locations were estimated by distributed linear inverse solutions based on a local autoregressive average (LAURA) (Grave de Peralta Menendez et al., 2001). LAURA estimates three-dimensional current density distributions using a realistic head model with a solution space of 4024 nodes equally distributed within the gray matter of the average template brain of the Montreal Neurological Institute (MNI). It makes no a priori assumptions regarding the number of sources or their locations and can deal with multiple simultaneously active sources (Michel et al., 2001). LAURA analyses were implemented using CARTOOL software by Denis Brunet. To ascertain the anatomical brain regions giving rise to the SSVEPs, the current source distributions estimated by LAURA were transformed into the standardized MNI coordinate system using SPM5 software (Wellcome Department of Imaging Neuroscience, London, UK).

\section{Results}

\section{Behavioral measures}

VGPs performed better than NVGPs in both speed and accuracy of target detections (Fig. 2) (supplemental Table 1, available at www.jneurosci.org as supplemental material). Detection accuracy $\left(d^{\prime}\right)$ was significantly higher in VGPs than in NVGPs over all three target types (number targets within the left and right letter sequences and "missing arm" targets at fixation $)\left(F_{(1,39)}=4.44\right.$, $p<0.05)$. There were no significant group $\times$ target type interactions, and no significant differences in performance were found between the left and right sequences at either the 8.6 or $12 \mathrm{~Hz}$
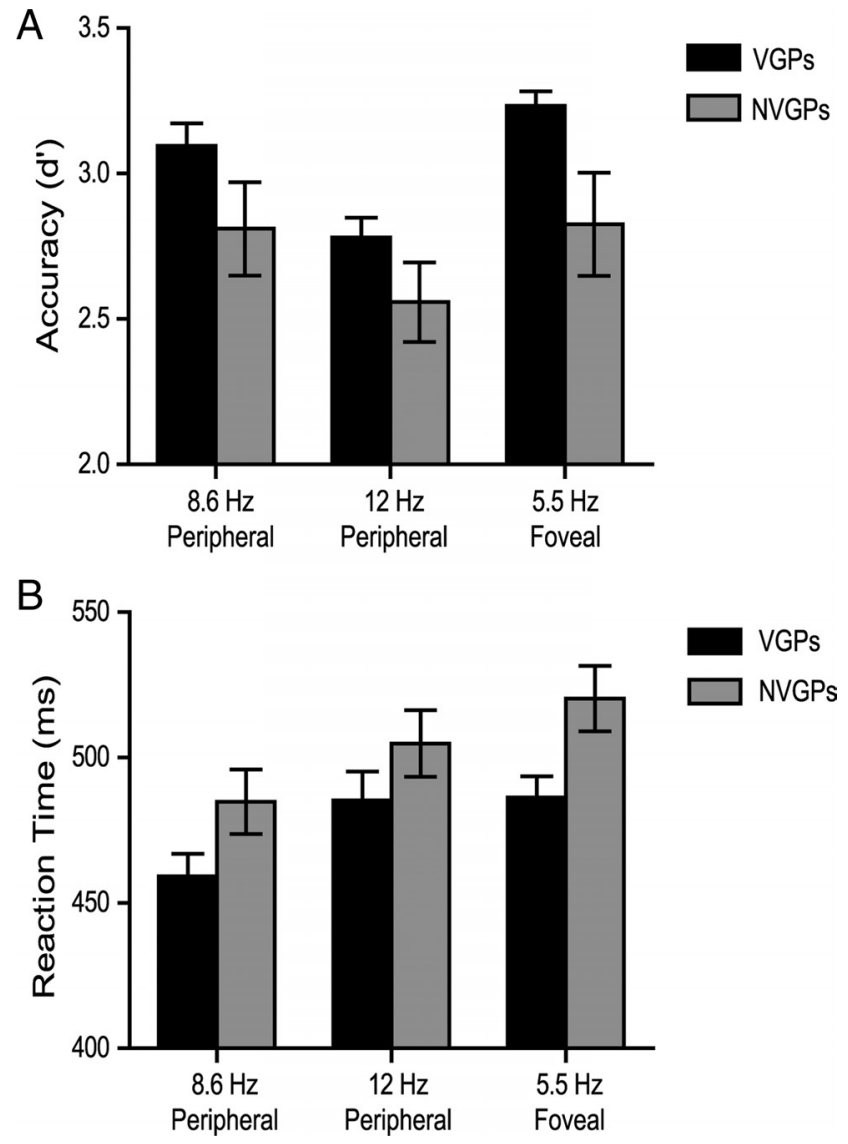

Figure 2. Behavioral performance of VGPs and NVGPs for detection of peripheral number targets within letter streams of 8.6 and $12 \mathrm{~Hz}$ and missing arm fixation cross targets. $A$, Detection accuracy $\left(d^{\prime}\right)$ measures. $\boldsymbol{B}$, Reaction times. Responses to the peripheral 8.6 and $12 \mathrm{~Hz}$ targets are averaged over left and right field presentations.

stimulus rate. Detection accuracy for number targets appearing within the $12 \mathrm{~Hz}$ letter sequence was significantly lower than for targets in the $8.6 \mathrm{~Hz}$ sequence in both groups $\left(F_{(1,39)}=17.16, p<\right.$ $0.0002)$. False alarm rates for the letter sequences (responses made outside the 200-1000 ms window after the attended targets) were low overall and somewhat higher in the NVGPs $(4.4 \%)$ than the VGPs $(1.9 \%)\left(t_{(39)}=2.11, p<0.05\right)$; additional analysis showed that false alarms were no more likely to occur after targets than after nontargets in the concurrent unattended letter sequence $\left(t_{(40)}=1.41, p>0.16\right)$, thereby indicating that the false alarms were not provoked by detections of targets in the unattended sequence.

Greater accuracy for target detection was accompanied by faster reaction times in VGPs versus NVGPs over all three target types $\left(F_{(1,39)}=4.66, p<0.04\right)$ (Fig. $2 B$ ), with no group $\times$ target type interactions. Paralleling the drop in detection accuracy for 12 versus $8.6 \mathrm{~Hz}$ peripheral targets, reaction times were also slower to the $12 \mathrm{~Hz}$ targets in both groups $\left(F_{(1,39)}=46.64, p<0.0001\right)$.

\section{SSVEP amplitudes}

The scalp topographies of the SSVEP amplitudes elicited by peripheral letter stimuli are shown in Figure 3. Because the asymmetry between contralateral and ipsilateral amplitudes did not differ between the left and right field stimulus sequences $\left(F_{(1,39)}=\right.$ $0.86, p>0.3)$, the topographies for the left and right field stimuli were averaged together in Figure 3, with the convention that 
A VGPs NVGPS
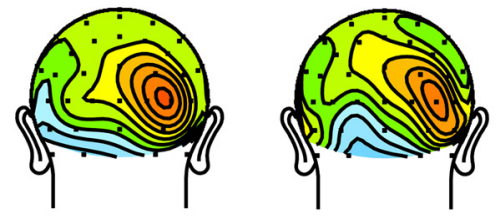

unAtt $8.6 \mathrm{~Hz}$ (Att $12 \mathrm{~Hz}$ Contralateral)
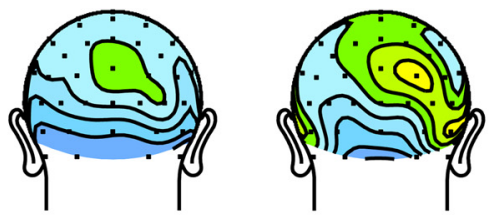

unAtt $8.6 \mathrm{~Hz}$ (Att $5.5 \mathrm{~Hz}$ Central)

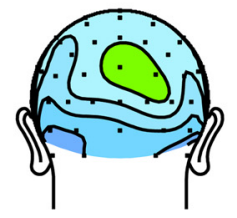

Ipsi Contra
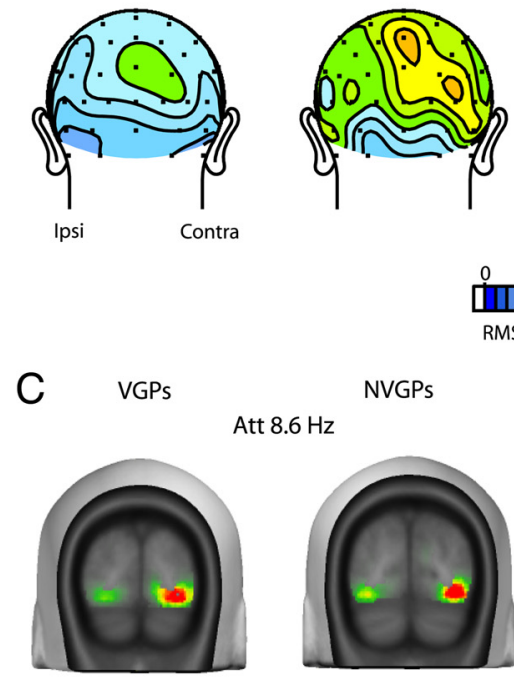
Att $8.6 \mathrm{~Hz}$

NVGPs

\section{RMS amplitude (uV) \\ $\stackrel{0}{\mathrm{~m}} \mathrm{~m} \mathrm{~m} \boldsymbol{\mathrm { m }} \mathrm{m}$}

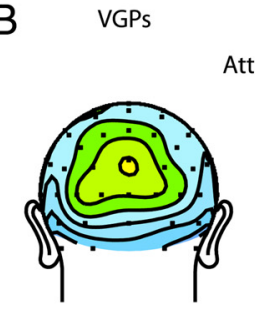

Att $12 \mathrm{~Hz}$

unAtt $12 \mathrm{~Hz}$ (Att $8.6 \mathrm{~Hz}$ Contralateral)

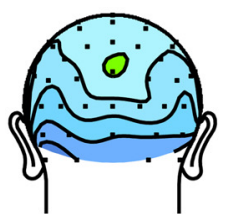

unAtt $12 \mathrm{~Hz}$ (Att $5.5 \mathrm{~Hz}$ Central)

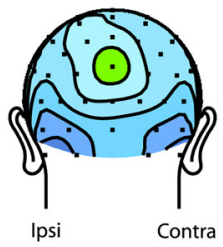

Contra

VGPS

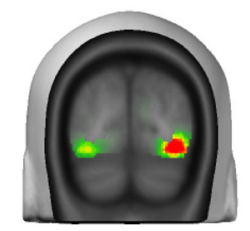

0

Figure 3. Topographical maps of SSVEP amplitudes elicited by peripheral letter sequences in VGPS and NVGPS. $\boldsymbol{A}$, Grand-averaged maps of SSVEP amplitudes (rms) to $8.6 \mathrm{~Hz}$ stimuli in VGPS ( $n=21$; left column) and NVGPs ( $n=20$; right column). Maps are shown for the attended condition (Att) (top row), unattended condition (unAtt) when attention was directed to the $12 \mathrm{~Hz}$ letter sequence in the opposite visual field (middle row), and unattended condition when attention was directed to the central $5.5 \mathrm{~Hz}$ stimuli (bottom row). Scalp topographies are averaged over left and right field stimulus sequences with the convention that contralateral (Contra) amplitudes are shown on the right of each head map and ipsilateral (Ipsi) amplitudes on the left. $\boldsymbol{B}$, Same as $\boldsymbol{A}$ for SSVEPs elicited by the peripheral $12 \mathrm{~Hz}$ stimuli. C, Estimated current sources for the grand-averaged attended SSVEPs corresponding to the scalp topographies shown in the top row. These inverse solutions were calculated using the LAURA algorithm and are given in units of current source density (nanoamperes per cubic millimeter).

contralateral sites are depicted on the right side of each scalp map and ipsilateral sites on the left. As seen in these scalp maps (Fig. $3 A, B)$ and in the bar graphs of averaged SSVEP amplitudes to the 8.6 and $12 \mathrm{~Hz}$ sequences (Fig. 4A) (supplemental Table 1, available at www.jneurosci.org as supplemental material), the attended letter sequences elicited SSVEPs of approximately the same amplitude in the two groups. The topographical distributions of the SSVEPs over the posterior scalp were also highly similar in the two groups, with strongly contralateral distributions at $8.6 \mathrm{~Hz}$ and more bilateral distributions at $12 \mathrm{~Hz}$.

The neural generators of the grand-averaged attended SSVEPs elicited in VGPs and NVGPs were modeled using a distributed minimum-norm linear inverse solution approach (LAURA). This analysis showed that the SSVEPs at both stimulation fre-
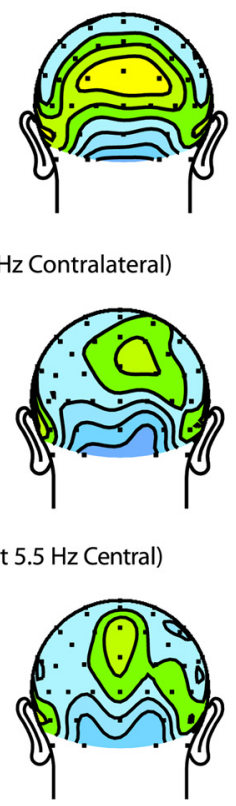

quencies ( 8.6 and $12 \mathrm{~Hz}$ ) could be accounted for by bilateral current sources in extrastriate visual cortex, with more of a contralateral dominance at $8.6 \mathrm{~Hz}$ (Fig. $3 C)$. These estimated source locations in ventrolateral occipital cortex (BA 19) were similar in the two groups. For the 8.6 $\mathrm{Hz}$ stimuli, the MNI coordinates of the peak of the source cluster were $\pm 30,-80$, -7 for VGPs and $\pm 36,-67,-5$ for NVGPs; for the $12 \mathrm{~Hz}$ stimuli, the coordinates were $\pm 33,-76,-8$ for VGPs and $\pm 36,-69,-7$ for NVGPs.

The effect of attention on SSVEP amplitudes was highly significant across both groups for the comparison of attended versus unattended peripheral letter sequences (Fig. $3 A, B$, top vs middle row) $\left(F_{(1,39)}=71.64, p<0.0001\right)$. The attention effect across both groups was also highly significant when comparing the SSVEPs with the attended versus unattended peripheral letter sequences when attention was directed to the central fixation stimuli (Fig. $3 A, B$, top vs bottom row $\left(F_{(1,39)}=20.80, p<0.0001\right)$.

The major difference between VGPs and NVGPs was evident in their SSVEP amplitudes to the unattended peripheral letter sequences, which were suppressed to a greater extent (relative to attended amplitudes) in the VGPs than in NVGPs. This suppression of the SSVEP to unattended (relative to attended) peripheral stimuli amounted to $32 \%$ in the VGPs and $15 \%$ in the NVGPs (attention $\times$ group interaction, $F_{(1,39)}=$ $4.36, p<0.05$ ) (Fig. 4A). This group difference in suppression of the unattended SSVEP was observed consistently both under conditions when the letter sequence in the opposite visual field was attended (VGPs, $32 \%$ and NVGPs, $17 \%$ ) and when attention was directed to central fixation (VGPs, 32\% and NVGPs, 13\%) [direction of attention $($ central/peripheral $) \times$ group interaction, $\left.F_{(1,39)}=0.52, p=\mathrm{NS}\right]$.

The effect of attention on the SSVEP amplitudes to the peripheral letter sequences was also quantified in each participant as an attentional modulation index: AMI = (attended - unattended)/(attended + unattended). Because unattended amplitudes did not differ when attention was directed to opposite-field stimuli versus central fixation stimuli, these amplitudes were averaged together in the AMI calculation. The AMI of the SSVEP, calculated for 8.6 and $12 \mathrm{~Hz}$ stimuli combined, was larger for the VGPs $(0.17)$ than for the NVGPs $(0.09)\left(t_{(39)}=1.86, p<0.04\right)$. These AMI values were also found to correlate significantly across subjects with speed of reaction time to peripheral targets $\left(r_{(39)}=-0.42, p<\right.$ $0.007)$, indicating faster reaction times in those subjects with the greatest suppression. The AMI-reaction time correlations were also significant for the 8.6 and $12 \mathrm{~Hz}$ sequences calculated separately $\left(8.6 \mathrm{~Hz}, r_{(39)}=-0.31, p<0.05 ; 12 \mathrm{~Hz}, r_{(39)}=\right.$ 
$-0.38, p<0.02)$. AMI correlations with detection accuracy $\left(d^{\prime}\right)$, however, were not significant.

The mean SSVEP amplitude to the central fixation square flashing at $5.5 \mathrm{~Hz}$ was larger than the mean amplitude to the peripheral alphanumeric sequences $\left(F_{(1,39)}=43.08, p<0.0001\right)$ (supplemental Table 1, available at www.jneurosci.org as supplemental material). The main effect of attention on the SSVEP amplitude to the central stimulus was significant across all subjects, with attended amplitudes being larger (attend-central vs attend-peripheral, $F_{(1,39)}=10.44, p<$ $0.003)$. The VGP and NVGP groups did not differ, however, in this attentional modulation of the SSVEP to the central stimulus (both the attention $\times$ group interaction and AMI comparison between groups were not significant) (Fig. $4 B$ ), nor were there any significant correlations between the AMI of the SSVEP to the central stimulus and detection accuracy or reaction times to central targets.

SSVEP amplitudes to the irrelevant $15 \mathrm{~Hz}$ letter sequence above fixation were also quantified. The $15 \mathrm{~Hz}$ SSVEP amplitudes did not differ between the attend-central versus attend-peripheral conditions, and no amplitude differences were found between the VGP and NVGP groups $\left(F_{(1,39)}=0.04, p>0.8\right)$ (Fig. $4 C$ ) (supplemental Table 1, available at www. jneurosci.org as supplemental material).

\section{ERPs to targets}

The ERPs elicited by the numerical targets in the attended peripheral letter sequences and the missing arm targets in the central $5.5 \mathrm{~Hz}$ stream had prominent P300 components peaking within the $450-470 \mathrm{~ms}$ interval (Fig. 5) (supplemental Table 1, available at www. jneurosci.org as supplemental material). The P300 amplitude (mean over 450-470 ms) was considerably smaller to targets in the peripheral 12 versus $8.6 \mathrm{~Hz}$ sequence $\left(F_{(1,39)}=61.46, p<\right.$ $0.0001)$, paralleling the reduced target detection accuracy at 12 $\mathrm{Hz}$ in both groups. $\mathrm{P} 300$ amplitudes were consistently reduced in NVGPs relative to VGPs: this group difference in P300 was significant in both the ERPs time-locked to the target stimuli (peripheral targets, $t_{(39)}=1.85, p<0.04$; central targets, $t_{(39)}=1.70$, $p<0.05$ ) and the ERPs time-locked to the button press signifying target detection (peripheral targets, $t_{(39)}=2.00, p<0.03$; central targets, $\left.t_{(39)}=2.62, p<.007\right)$. This finding of reduced P300 amplitudes in NVGPs in both stimulus-locked and responselocked averages indicates that the group difference was not a simple consequence of temporal dispersion of the waveform because of greater reaction time variability in the NVGPs.

There were no significant differences in P300 latency between the VGPs versus NVGPs for either the peripheral targets (458 vs $464 \mathrm{~ms}$ ) or the central targets ( 456 vs $453 \mathrm{~ms}$ ). Finally, P300s were absent or greatly reduced in the ERPs to unattended peripheral targets in both VGPs and NVGPs (Fig. 5B) (attention effect on
B

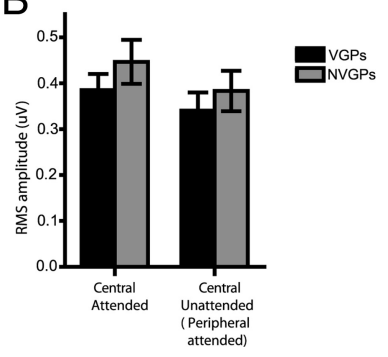

C

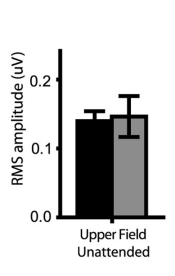
VVPs

Figure 4. Mean amplitudes of SSVEPs to the different stimulus sequences in VGPs and NVGPS. $A$, Attended and unattended amplitudes to peripheral sequences ( 8.6 and $12 \mathrm{~Hz}$ combined); SSVEPs to the unattended letter sequences were suppressed to a greater extent in VGPs than in NVGPS. B, SSVEPs to central $(5.5 \mathrm{~Hz})$ stimuli were similarly increased by attention in the two groups. C, SSVEPs to the always irrelevant $15 \mathrm{~Hz}$ letter sequence in the upper field did not differ between groups.
A

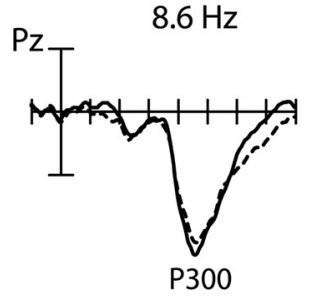

Peripheral Unattended Targets

$12 \mathrm{~Hz}$

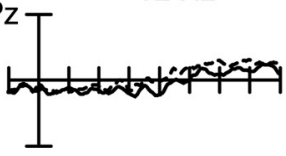

Central Attended Targets
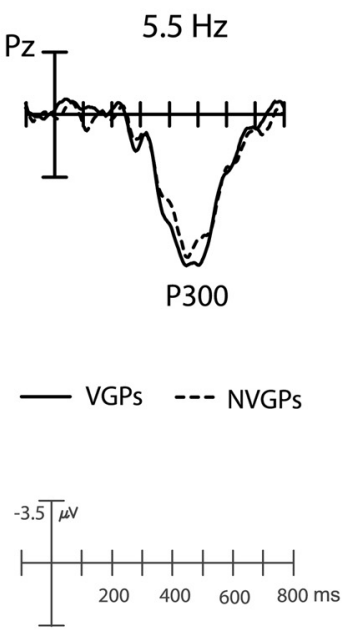

Figure 5. ERPs to infrequent number targets in peripheral 8.6 and $12 \mathrm{~Hz}$ alphanumeric sequences and to central fixation targets in VGPs and NVGPS. $\boldsymbol{A}$, ERPs to attended targets. $\boldsymbol{B}$, ERPs to unattended peripheral targets. Waveforms shown are time-locked to target presentations. Response-locked waveforms described in text are not shown.

P300 amplitude, $\left.F_{(1,39)}=215.06, p<0.0001\right)$. No unattended targets were presented at central fixation.

\section{Discussion}

The present study reinforces previous behavioral findings that have demonstrated superior performance capabilities of VGPs versus NVGPs in a variety of attention-demanding tasks (Greenfield et al., 1994; Green and Bavelier, 2003, 2006b; Cohen et al., 2007; Feng et al., 2007; Spence et al., 2009; Hubert-Wallander et al., 2010). These previous studies established a strong link between action game play and enhanced spatial and temporal visual selective attention. The current task of detecting targets in RSVP sequences was designed to manipulate both spatial and temporal aspects of selective visual attention and to probe the neural bases of the enhanced capabilities of VGPs. Behaviorally, we found that VGPs detected targets in rapidly flashing sequences in both the central and peripheral visual fields with greater speed and accuracy than NVGPs. Electrophysiological recordings of SSVEPs to the flashing stimuli provided insight into whether this attentional superiority of the VGPs was based on a mechanism of signal enhancement or distractor suppression. Whereas the SSVEPs elicited in the visual cortex by attended alphanumeric sequences were of similar amplitude in the two groups, the VGPs showed a greater suppression of SSVEPs to these rapidly flashed sequences 
when attention was directed elsewhere. Thus, an enhanced ability to suppress the cortical processing of irrelevant, distracting visual information may facilitate target detection performance in the VGPs. Differences were also observed between VGPs and NVGPs in their ERPs to target stimuli in the rapidly flashing sequences. The P300 component was larger in amplitude to attended targets in the VGPs than NVGPs, which is consistent with action VGP superiority in perceptual discrimination and decision processes (Hillyard and Picton 1987; Polich, 2007).

Previous studies have demonstrated that the frequencytagged SSVEP is a sensitive index of the allocation of visual attention to repetitive visual stimuli over a wide range of stimulation rates and task conditions (Müller et al., 1998a,b, 2003, 2006; Di Russo and Spinelli, 1999; Müller and Hillyard, 2000; Di Russo et al., 2001, 2002; Müller and Hubner, 2002; Chen et al., 2003; Kelly et al., 2005; Ding et al., 2006; Talsma et al., 2006; Kim et al., 2007; Andersen et al., 2008, 2009; Toffanin et al., 2009). These studies have generally found that an attended flickering stimulus elicits a larger SSVEP than the same stimulus when unattended, thus providing evidence that attention modulates the sensory gain of visual inputs at the level of the visual cortex. In the current study, VGPs differed from NVGPs in their ability to suppress the SSVEP to unattended peripheral stimulus sequences. The neural generators of the attention-related SSVEPs were estimated to lie in ventral lateral extrastriate visual cortex, and the modulations of SSVEP amplitude with attention were found to be strongly correlated across subjects with the reaction times to targets. These findings suggest that the behavioral advantage of the VGPs in detecting targets under high load conditions was, at least in part, attributable to their ability to suppress the irrelevant streams of sensory input at the level of extrastriate visual cortex.

The enhanced suppression of irrelevant sensory input in VGPs, however, was only observed for the peripheral alphanumeric sequences. Attentional modulation of the SSVEP to the flashing central stimuli was equivalent in the two groups. This may reflect a difference in the degree to which central and peripheral inputs can be modulated by attention (Beck and Lavie, 2005; Handy and Khoe, 2005; Ruff et al., 2006) (but see Frey et al., 2010). The central-peripheral difference in SSVEP modulation observed here is difficult to interpret, however, because the central and peripheral sequences differed in several respects, including flicker rate $(8.6 / 12$ vs $5.5 \mathrm{~Hz})$, stimulus type (fixation cross and square vs alphanumeric sequence), and target frequency of occurrence (every 5.7 vs $3.75 \mathrm{~s}$ ). Because of these substantial differences between the central and peripheral sequences, we cannot reach a conclusion as to whether VGPs are generally more adept than NVGPs at suppressing irrelevant peripheral as opposed to central inputs.

Although the present study did not have a training manipulation that compared pretraining and posttraining behavioral and neural measures in non-game-playing controls, the results obtained here should be interpreted in the context of several previous investigations that did demonstrate a causal link between action videogame playing and enhanced selective attention capabilities. In particular, more than half a dozen training studies document the causal role of action game play in improved attentional selection in both space and time (spatial selection: Green and Bavelier, 2003, 2006a, 2007; Feng et al., 2007; Spence et al., 2009; temporal selection: Green and Bavelier, 2003; Cohen et al., 2007; Li et al., 2010). In the context of this expanding evidence in the literature, it seems most likely that the group differences noted in the present study are not simply a consequence of selfselection sampling bias but represent attentional skills that are trainable by action game play. The SSVEP evidence presented here advances our understanding by showing that the behavioral superiority of VGPs was associated with greater distractor suppression in the visual periphery. This enhanced ability to suppress distracting input in action game players provides a striking contrast to recent studies suggesting that heavy users of media technologies may suffer, on the contrary, from greater distractibility and from problems in focusing attention (Ophir et al., 2009; Swing et al., 2010).

Under the present high load conditions, the P300 component of the ERPs to the number targets in the rapidly flashing alphanumeric sequences was found to be larger in the VGPs than in the NVGPs. Several decades of research on the P300 have shown that this component is specifically elicited by the delivery of taskrelevant information that occurs unpredictably (for review, see Kutas et al., 1977; Hillyard and Picton, 1987; Picton, 1992; Polich, $1998,2007)$. These studies have found that the latency of the P300 provides an index of the timing of perceptual discrimination and decision processes, whereas its amplitude varies with the informational content of the eliciting stimulus and the confidence of the perceptual decision. Although the P300 peak latencies did not differ between groups in the present study, the larger amplitudes produced by the VGPs in the peak latency range suggests that the VGPs can make perceptual decisions more accurately and confidently under high load conditions. Furthermore, the finding that targets in the unattended alphanumeric sequence did not elicit appreciable P300 components (nor behavioral false alarm responses) in either VGPs or NVGPs provides additional evidence that attention was selectively focused on the attended stimulus sequence in both groups. Using a very different paradigm, Green et al. (2010) have also recently concluded that VGPs exhibit higher perceptual sensitivity, which allows them to make more informed decisions. A training study established the causal effect of action game playing on this change in sensitivity, ruling out subject selection biases as an explanation for improved performance. The present study provides converging evidence that VGPs are superior at making sensory discriminations that facilitate perceptual decision making.

In summary, the present study examined the neural basis of the performance superiority of VGPs relative to NVGPs in an attention-demanding task that required detection of targets presented in rapid sequences. Our electrophysiological recordings pointed to two mechanisms that may underlie this superiority. The first was an enhanced ability to suppress irrelevant distracting information, which presumably would reduce interference with the execution of the primary task. The second was superior ability to make accurate discriminations and perceptual decisions under high load conditions. When interpreted in the context of several previous behavioral studies that have convincingly demonstrated a causal link between action videogame training and attentional enhancements, the differences observed here between VGPs and NVGPs can most plausibly be attributed to videogame playing experience rather than to an initial predisposition and self-selection. The current findings that videogame playing is associated with modification of the neural circuitry for both sensory input control and perceptual decision making suggest the utility of such games as a training intervention to enhance attentional control and resistance to distraction (Achtman et al., 2008; Green and Bavelier, 2008).

\section{References}

Achtman RL, Green CS, Bavelier D (2008) Video games as a tool to train visual skills. Restor Neurol Neurosci 26:435-446. 
Andersen SK, Hillyard SA, Müller MM (2008) Attention facilitates multiple stimulus features in parallel in human visual cortex. Curr Biol 18: $1006-1009$.

Andersen SK, Muller MM, Hillyard SA (2009) Color-selective attention need not be mediated by spatial attention. J Vis 9:2.1-2.7.

Beck DM, Lavie N (2005) Look here but ignore what you see: effects of distractors at fixation. J Exp Psychol Hum Percept Perform 31:592-607.

Boot WR, Kramer AF, Simons DJ, Fabiani M, Gratton G (2008) The effects of video game playing on attention, memory and executive control. Acta Psychologica 129:387-398.

Castel AD, Pratt J, Drummond E (2005) The effects of action video game experience on the time course of inhibition of return and the efficiency of visual search. Acta Psychol (Amst) 119:217-230.

Chen Y, Seth AK, Gally JA, Edelman GM (2003) The power of human brain magnetoencephalographic signals can be modulated up or down by changes in an attentive visual task. Proc Natl Acad Sci U S A 100: 3501-3506.

Chisholm JD, Hickey C, Theeuwes J, Kingstone A (2010) Reduced attentional capture in action video game players. Atten Percept Psychophys 72:667-671.

Cohen JE, Green CS, Bavelier D (2007) Training visual attention with video games: not all games are created equal. In: Computer games and team and individual learning (O’Neil H, Perez R, eds), pp 205-227. Amsterdam: Elsevier.

Ding J, Sperling G, Srinivasan R (2006) Attentional modulation of SSVEP power depends on the network tagged by the flicker frequency. Cereb Cortex 16:1016-1029.

Di Russo F, Spinelli D (1999) Spatial attention has different effects on the magno- and parvocellular pathways. Neuroreport 10:2755-2762.

Di Russo F, Spinelli D, Morrone MC (2001) Automatic gain control contrast mechanisms are modulated by attention in humans: evidence from visual evoked potentials. Vision Res 41:2435-2447.

Di Russo F, Teder-Sälejärvi W, Hillyard SA (2002) Steady-state VEP and attentional visual processing. In: The cognitive electrophysiology of mind and brain (Zani A, Proverbio AM, eds), pp 259-274. New York: Academic.

Dye MW, Green CS, Bavelier D (2009) The development of attention skills in action video game players. Neuropsychologia 47:1780-1789.

Feng J, Spence I, Pratt J (2007) Playing an action video game reduces gender differences in spatial cognition. Psychol Sci 18:850-855.

Frey HP, Kelly SP, Lalor EC, Foxe JJ (2010) Early spatial attentional modulation of inputs to the fovea. J Neurosci 30:4547-4551.

Grave de Peralta Menendez R, Gonzalez Andino S, Lantz G, Michel CM, Landis T (2001) Noninvasive localization of electromagnetic epileptic activity. I. Method descriptions and simulations. Brain Topogr 14:131-137.

Green CS, Bavelier D (2003) Action video game modifies visual selective attention. Nature 423:534-537.

Green CS, Bavelier D (2006a) Enumeration versus multiple object tracking: the case of action video game players. Cognition 101:217-245.

Green CS, Bavelier D (2006b) Effect of action video games on the spatial distribution of visuospatial attention. J Exp Psychol Hum Percept Perform 32:1465-1478.

Green CS, Bavelier D (2007) Action-video-game experience alters the spatial resolution of vision. Psychol Sci 18:88-94.

Green CS, Bavelier D (2008) Exercising your brain: a review of human brain plasticity and training-induced learning. Psychol Aging 23:692-701.

Green CS, Pouget A, Bavelier D (2010) A general mechanism for learning with action video games: improved probabilistic inference. Curr Biol 20:1573-1579.

Greenfield PM, DeWinstanley P, Kilpatrick H, Kaye D (1994) Action video games and informal education: effects on strategies for dividing visual attention. J Appl Dev Psychol 15:105-123.

Handy TC, Khoe W (2005) Attention and sensory gain control: a peripheral visual process? J Cogn Neurosci 17:1936-1949.

Hillyard SA, Picton TW (1987) Electrophysiology of cognition. In: Handbook of physiology, Sec 1, The nervous system, Vol V, Higher functions of the brain, Pt 2 (Plum F, ed), pp 519-584. Bethesda, MD: American Physiological Society.

Hubert-Wallander B, Green CS, Bavelier D (2010) Stretching the limits of visual attention: the case of action video games. Wiley interdisciplinary reviews: cognitive science, n/a. doi: 10:1002/wcs.116.

Kelly SP, Lalor EC, Reilly RB, Foxe JJ (2005) Visual spatial attention tracking using high-density SSVEP data for independent brain-computer communication. IEEE Trans Neural Syst Rehabil Eng 13:172-178.

Kim YJ, Grabowecky M, Paller KA, Muthu K, Suzuki S (2007) Attention induces synchronization-based response gain in steady-state visual evoked potentials. Nat Neurosci 10:117-125.

Kutas M, McCarthy G, Donchin E (1977) Augmenting mental chronometry: the P300 as a measure of stimulus evaluation time. Science 197:792-795.

Li R, Polat U, Scalzo F, Bavelier D (2010) Reducing backward masking through action video game training. J Vis 10:33.

Macmillan NA, Creelman CD (1991) Detection theory: a user's guide. New York: Cambridge UP.

Michel CM, Thut G, Morand S, Khateb A, Pegna AJ, Grave de Peralta R, Gonzalez S, Seeck M, Landis T (2001) Electric source imaging of human brain functions. Brain Res Brain Res Rev 36:108-118.

Müller MM, Hillyard S (2000) Concurrent recording of steady-state and transient event-related potentials as indices of visual-spatial selective attention. Clin Neurophysiol 111:1544-1552.

Müller MM, Hübner R (2002) Can the spotlight of attention be shaped like a doughnut? Evidence from steady-state visual evoked potentials. Psychol Sci 13:119-124

Müller MM, Teder-Sälejärvi W, Hillyard SA (1998a) The time course of cortical facilitation during cued shifts of spatial attention. Nat Neurosci 1:631-634.

Müller MM, Picton TW, Valdes-Sosa P, Riera J, Teder-Sälejärvi WA, Hillyard SA (1998b) Effects of spatial selective attention on the steady-state visual evoked potential in the $20-28 \mathrm{~Hz}$ range. Brain Res Cogn Brain Res 6:249-261.

Müller MM, Malinowski P, Gruber T, Hillyard SA (2003) Sustained division of the attentional spotlight. Nature 424:309-312.

Müller MM, Andersen S, Trujillo NJ, Valdés-Sosa P, Malinowski P, Hillyard SA (2006) Feature-selective attention enhances color signals in early visual areas of the human brain. Proc Natl Acad Sci USA 103:14250-14254.

Ophir E, Nass C, Wagner AD (2009) Cognitive control in media multitaskers. Proc Natl Acad Sci U S A 106:15583-15587.

Picton TW (1992) The P300 wave of the human event-related potential. J Clin Neurophysiol 9:456-479.

Polich J (1998) P300 clinical utility and control of variability. J Clin Neurophysiol 15:14-33.

Polich J (2007) Updating P300: an integrative theory of P3a and P3b. Clin Neurophysiol 118:2128-2148.

Ruff CC, Blankenburg F, Bjoertomt O, Bestmann S, Freeman E, Haynes JD, Rees G, Josephs O, Deichmann R, Driver J (2006) Concurrent TMSfMRI and psychophysics reveal frontal influences on human retinotopic visual cortex. Curr Biol 16:1479-1488.

Spence I, Yu JJ, Feng J, Marshman J (2009) Women match men when learning a spatial skill. J Exp Psychol Learn Mem Cogn 35:1097-1103.

Swing EL, Gentile DA, Anderson CA, Walsh DA (2010) Television and video game exposure and the development of attention problems. Pediatrics 126:214-221.

Talsma D, Doty TJ, Strowd R, Woldorff MG (2006) Attentional capacity for processing concurrent stimuli is larger across sensory modalities than within a modality. Psychophysiology 43:541-549.

Teder-Sälejärvi WA, Di Russo F, McDonald JJ, Hillyard SA (2005) Effects of spatial congruity on audio-visual multimodal integration. J Cogn Neurosci 17:1396-1409.

Toffanin P, de Jong R, Johnson A, Martens S (2009) Using frequency tagging to quantify attentional deployment in a visual divided attention task. Int J Psychophysiol 72:289-298.

Vialatte FB, Maurice M, Dauwels J, Cichocki A (2010) Steady-state visually evoked potentials: focus on essential paradigms and future perspectives. Prog Neurobiol 90:418-438.

West GL, Stevens SA, Pun C, Pratt J (2008) Visuospatial experience modulates attentional capture: evidence from action video game players. J Vis 8:13.1-13.9. 